\title{
Avaliação dos atributos da Atenção Primária à Saúde num município do interior do Estado de São Paulo - Brasil
}

\section{Evaluation of the Primary Healthcare attributes in a municipality located in the countryside of São Paulo State - Brazil}

\author{
Lislaine Aparecida Fracolli* \\ Marianne Junko Muramatsu** \\ Maria Fernanda Pereira Gomes*** \\ Fabiana Rodrigues Zequini Nabão****
}

\begin{abstract}
Resumo
A Atenção Primária à Saúde pode ser qualificada pela existência de quatro atributos essenciais: acesso de primeiro contato do indivíduo com o sistema de saúde, longitudinalidade, integralidade, coordenação da atenção e três derivados: orientação familiar, orientação comunitária e competência cultural. Nesta perspectiva o objetivo desta pesquisa foi avaliar a presença e a extensão dos atributos da Atenção Primária à Saúde (APS) na Estratégia Saúde da Família (ESF) no município de Quatá-Sp. Trata-se de uma pesquisa avaliativa e quantitativa, os dados foram coletados por meio de entrevistas com 34 cuidadores de crianças menores de dois anos utilizando o instrumento PCATool versão criança no mês de janeiro de 2014. Os atributos da APS apresentam-se no geral como satisfatórios, no entanto, a acessibilidade e os serviços disponíveis precisam melhorar sua operacionalização. Nesta pesquisa identificou-se a necessidade de melhorar o acesso da população aos serviços de APS, com possíveis adequações a serem realizadas no horário de funcionamento das unidades de saúde; facilitar o acesso e o agendamento de consultas; melhorar o sistema de referência e contrarreferência; disponibilidade de serviços como vacinas, sutura; e melhorar a comunicação entre profissionais e usuários, revisando e reformulando o modelo assistencial da ESF.
\end{abstract}

Palavras-chave: Atenção Primária à Saúde. Serviços de Saúde Infantil. Avaliação em Saúde.

\begin{abstract}
The Primary Healthcare can be qualified by the existence of four main attributes: access of the individual's first contact with the healthcare system, longitudinality, integrality, coordination of care and three derivatives: family counseling, community orientation and cultural competence. Therefore, this study aimed to evaluate the existence and the extent of the Primary Healthcare attributes (PH) in the Family Health Strategy (FHS) in the municipality of Quatá-SP. The evaluative and quantitative study collected data through interviews with 34 caregivers who have cared for children under two years old by the use of PCATool child version in January 2014. The Primary Healthcare attributes have been reported as satisfactory; however, the accessibility and available services should improve their workability. This study identified the necessity to improve the population's access to the Primary Healthcare services. Proper adaptations should be performed during the opening hours of the healthcare centers; facilitating access and appointment scheduling; improve the counter-reference and reference system; offer vaccination and stitch services; and also, improve the communication between professionals and users, review and reformulate the attendance model of the FHS
\end{abstract}

Keywords: Primary Healthcare. Child Health Services. Health Evaluation.

DOI: 10.15343/0104-7809.201539015461

* Universidade de São Paulo. São Paulo - SP, Brasil. E-mail: lislaine@usp.br

** Escola de Enfermagem da Universidade de São Paulo. São Paulo - SP. Brasil. E-mail: mariannejmt@gmail.com

*** Escola de Enfermagem da Universidade de São Paulo. Marília-SP, Brasil. E-mail: mferpg@usp.br

**** Universidade Paulista Assis. Assis-SP, Brasil. E-mail: frzequini@gmail.com

Os autores declaram não haver conflitos de interesse. 


\section{INTRODUÇÃO}

A Atenção Primária à Saúde (APS) representa o primeiro nível de contato dos indivíduos, da família e da comunidade com o sistema nacional de saúde, pelo qual os cuidados de saúde são levados, o mais proximamente possível aos lugares onde pessoas vivem e trabalham, e constituem o primeiro elemento de um continuado processo de assistência à saúde ${ }^{1}$.

Nesta perspectiva é possível caracterizar a Estratégia da Saúde da Família (ESF) como uma estratégia de implantação de equipes de Atenção Primária à Saúde (APS). A ESF tem como objetivo reorientar a prática assistencial em direção a uma assistência à saúde centrada na família, entendida e percebida a partir de seu ambiente físico e social ${ }^{2}$.

Nesse contexto, a ESF tem contribuído significativamente para o aumento do acesso das pessoas aos serviços de saúde, e para a aproximação da equipe de saúde com as condições de vida e saúde das pessoas, tem também corroborado para resultados positivos nos indicadores de saúde. Com base no impacto que a ESF tem demonstrado na APS é importante avaliá-la com a finalidade de apontar as suas potencialidades e fragilidades que podem servir como subsídio para gestão em saúde.

A avaliação é uma atividade que consiste em fazer o julgamento sobre uma determinada intervenção, ou sobre qualquer um de seus componentes com o intuito de auxiliar na tomada de decisões ${ }^{3}$.

A Atenção Primária à Saúde, proposta por Starfield ${ }^{4}$ pode ser avaliada pela existência de quatro atributos essenciais: acesso de primeiro contato do indivíduo com o sistema de saúde, longitudinalidade, integralidade, coordenação da atenção; e três atributos derivados: orientação familiar, orientação comunitária e competência cultural.

Com isso, avaliar os serviços de saúde envolve a produção de conhecimento e/ou instrumentos visando à melhoria da assistência prestada através das técnicas e tecnologias desenvolvidas pelos profissionais de saúde $\mathrm{e}^{5,6}$.
Com base na importância de avaliar a ESF, escolheu-se o instrumento de avaliação validado no Brasil, Primary Care Assessment Tool (PCATool) versão criança para avaliar a APS disponível para as crianças menores de dois anos de idade. A escolha do PCATool, partiu da análise que este instrumento possui a avaliação dos atributos: orientação familiar e orientação comunitária, atributos pilares da ESF.

O município de Quatá-SP, foi escolhido para desenvolver está pesquisa por se tratar de um município de pequeno porte com 100\% de cobertura de ESF e Índice Paulista de Responsabilidade Social (IPRS) igual a 4 caracterizando este município como de baixa riqueza e de níveis intermediários de longevidade e/ou escolaridade. Nesta perspectiva espera-se que a saúde da criança no município de Quatá tenha alto grau de orientação para APS, por apresentar 100\% de cobertura deste tipo de serviço.

Partindo do pressuposto de que é importante avaliar a ESF, o objetivo desta pesquisa foi avaliar a presença e a extensão dos atributos da APS na perspectiva dos cuidadores de crianças menores de dois anos que utilizam a Estratégia Saúde da Família implantada no município de Quatá-SP, por meio da aplicação do PCATool Versão Criança.

\section{METODOLOGIA}

Trata-se de uma pesquisa avaliativa e descritiva, realizada no município de Quatá, interior do estado de São Paulo, na região de Presidente Prudente. O município de Quatá possui 12972 habitantes com 100\% de cobertura de ESF e 4 equipes de saúde da família. Foram sujeitos desta pesquisa 34 cuidadores de crianças menores de 2 anos residentes na área de atuação de uma das equipes de ESF do município. Para esta pesquisa foi utilizado os critérios de inclusão para os sujeitos: Ser cuidador, de criança menor de 02 anos de idade; e a criança e o cuidador devem morar na área da ESF selecionada há mais de um ano ${ }^{7}$.

Utilizou-se como instrumento de coleta de dados o PCATool Versão Criança, instrumento aprovado pelo Ministério da Saúde. O PCATool 
permite, por meio de entrevistas domiciliares ou em serviços de saúde aplicadas por entrevistadores treinados, identificar aspectos da estrutura e processo dos serviços que exigem reafirmação ou reformulação na busca da qualidade, tanto para o planejamento como para a execução das ações de APS $^{8}$.

Para a aplicação do PCATool versão criança, foi utilizada a ajuda voluntária dos Agentes Comunitários de Saúde (ACS) que trabalhavam na ESF selecionada para a coleta de dados. Os ACS tiveram a orientação necessária para realizarem a pesquisa. A pesquisa foi realizada no mês de janeiro de 2014.

O PCATool, foi disponibilizado aos participantes após o consentimento do secretário de saúde do município de Quatá e após a aprovação do Comitê de Ética e Pesquisa, sob o número de parecer: 489977.

Para a organização dos dados foi elaborado um banco de dados no software Microsoft

Fonte: Elaborado pelos autores.
Excel. E para a análise estatística dos dados foi utilizado o Software Statistical Package for Social Sciences (SPSS) versão 17.0, foi também utilizado o intervalo de confiança de 95\% e a aplicação do teste estatístico paramétrico Teste T. O serviço estatístico da Escola de Enfermagem da Universidade de São Paulo foi consultado para a organização e análise dos dados.

\section{RESULTADOS}

Os sujeitos entrevistados, foram mães, pais e outros cuidadores, principais responsáveis pelas crianças menores de 2 anos de idade. Foram entrevistadas 34 famílias cadastradas na ESF selecionada. Não houve nenhuma recusa à pesquisa.

O quadro 1 mostra os escores dos atributos essenciais e derivados, o desvio padrão (DP), teste t, valor mínimo e valor máximo.

Quadro 1. Valores dos escores dos atributos da APS na ESF II, Quatá, 2013.

\begin{tabular}{lccccc}
\hline Atributo & Escore & DP & Teste t & Mín. & Máx. \\
\hline Grau de Afiliação & 9,31 & 0,48 & 1.000 & 2,00 & 4,00 \\
Acesso de Primeiro Contato - Utilização & 9,22 & 1,14 & 1,000 & 5,56 & 10,0 \\
Acesso de Primeiro Contato - Acessibilidade & 6,75 & 1,49 & 0,717 & 3,89 & 10,0 \\
Longitudinalidade & 8,54 & 0,98 & 1,000 & 5,48 & 10,0 \\
Coordenação - Integração de Cuidados & 7,60 & 4,28 & 0,685 & 0,00 & 10,0 \\
Sistema de Informaç̃̃es & 8,89 & 0,91 & 1,000 & 6,67 & 10,0 \\
Integralidade - Serviços Disponíveis & 6,78 & 0,8 & 0,898 & 4,81 & 8,15 \\
Integralidade- Serviços Prestados & 9.39 & 1,19 & 1,000 & 6,00 & 10,0 \\
Orientação Familiar & 7,29 & 2,21 & 0,961 & 0,00 & 10,0 \\
Orientação Comunitária & 7,72 & 1,09 & 1,000 & 5,83 & 10,0 \\
Essencial & 7,62 & 0,56 & 1,000 & 6,15 & 8,67 \\
Geral & 7,60 & 0,68 & 1,000 & 6,24 & 8,92 \\
\hline
\end{tabular}

Os resultados mostram que de forma geral o munícipio de Quatá possui orientação para APS, pois apresenta Escore Geral dos atributos igual a 7,60 e Escore Essencial igual a 7,62. Nesta pesquisa destacaram-se os atributos de Integralidade - Serviços Prestados com escore igual a 9,39, serviços que são executados na prática pelas ESFs;
Grau de Afiliação com escore igual 9,31, indicando que costuma ser o mesmo profissional que acompanha as crianças; Acesso de Primeiro Contato - Utilização com escore igual 9,22, este atributo mostra que a Saúde da Família é a primeira escolha dos cuidadores para levarem a criança quando necessita de atendimento de saúde. 
Observa-se também que o atributo de Longitudinalidade é bem avaliado pelos cuidadores de crianças apresentando escore igual a 8,54. Os atributos de Acesso de Primeiro Contato Acessibilidade com escore igual a 6,75 e Integralidade - Serviços Disponíveis com escore igual a 6,78 precisam melhorar sua operacionalização no município de Quatá.

\section{DISCUSSÃO}

De acordo com Starfield ${ }^{4}$, considera-se o valor 6,6 o mínimo para o serviço de saúde ter a presença do atributo em seu serviço de acordo com a avaliação da família das crianças atendidas. Nessa perspectiva utilizamos o valor 6,6 como valor de referência para analisar os escores dos atributos, bem como os escores essencial e geral.

No atributo Grau de Afiliação, encontramos escore maior que o valor de referência. Foi observado que do total de entrevistados, 100\% $(\mathrm{N}=34)$ responderam a existência de um serviço de saúde em que "geralmente" levam a criança, quando a mesma necessita de atendimento.

Em relação ao atributo Grau de Afiliação, quando perguntamos aos entrevistados se existia um serviço de saúde que "melhor conhecia" a criança, $88,23 \%(N=30)$ disseram que sim e que é o mesmo médico que os cuidadores geralmente levam a criança; $8,82 \%(\mathrm{~N}=3)$ disseram que o serviço de saúde que melhor conhece a criança não é o serviço em que a criança geralmente é levada quando necessita de atendimento.

Quando os entrevistados foram questionados se havia um médico ou serviço "mais responsável" pelo atendimento de saúde da criança, $11,76 \%(\mathrm{~N}=4)$ responderam ser $\mathrm{o}$ mesmo serviço de saúde "que melhor conhece" a criança; 2,94\% $(\mathrm{N}=1)$ respondeu ser aquele serviço em que "geralmente leva" a criança; $2,94 \%(N=1)$ respondeu não haver um serviço "mais responsável" e 82,35\% (N=28) responderam ser o mesmo serviço que "geralmente leva" e que "melhor conhece" a criança.

O atributo de Primeiro Contato, é dividido em 2 tópicos: acessibilidade e utilização do serviço de saúde como fonte de cuidado a cada novo problema ou novo episódio de um mesmo problema de saúde, com exceção das verdadeiras emergências e urgências médicas ${ }^{8}$.

O escore de Primeiro Contato referente à utilização do serviço apresentou escore maior que o valor de referência. Ao perguntar aos entrevistados sobre o primeiro serviço procurado para uma consulta da criança, 25 (73,52\%) dos entrevistados responderam que a ESF "com certeza" é o primeiro serviço de saúde a ser procurado; 28 (82,35\%) disseram que procuram a ESF quando a criança apresenta um novo problema de saúde e 31 (91,17\%) responderam que o serviço encaminha as crianças quando necessitam de atendimento especializado.

Em relação ao atributo Acesso de Primeiro Contato - Acessibilidade, o escore se apresentou igual a 6,75, maior do que o valor de referência, porém com valor aproximado. Observou-se que os entrevistados esperam mais que 30 minutos pela consulta médica e apresentam dificuldade para obter aconselhamento da ESF via contato telefônico.

O valor alto do atributo acesso de primeiro contato - utilização demonstra que os usuários identificam o serviço de saúde como referência ao cuidado infantil. A unidade de ESF constitui o primeiro local a ser procurado pelos cuidadores quando a criança necessita de cuidados de saúde.

A acessibilidade é um dos elementos estruturais da APS, envolve a localização próxima à população, horários e dias abertos para atender, o grau de tolerância para consultas não agendadas e o quanto a população percebe estes aspectos da acessibilidade. A acessibilidade implica no acesso e uso do serviço a cada novo problema ou novo episódio de um problema pelo qual as pessoas buscam atenção à saúde ${ }^{4,9}$.

No atributo de primeiro contato - acessibilidade, identificamos que há dificuldades no acesso, pois, é possível conseguir uma consulta no mesmo dia, porém, os usuários tem que chegar no horário de abertura da unidade de saúde. Os entrevistados também referem que para agendar uma consulta a espera na recepção é 
muito grande, e que depois de terem agendado a consulta, o atendimento demora mais que 15 dias para acontecer, além da dificuldade em obter um atendimento por via telefônica.

O atributo Longitudinalidade teve escore igual a 8,54, este valor é considerado alto em relação ao valor de referência Este atributo de acordo com Starfield ${ }^{4}$ pressupõe a existência de uma fonte regular de atenção e seu uso ao longo do tempo. Sendo este atributo, relativo ao vínculo da população com sua fonte de atenção, evidencia-se os fortes laços interpessoais que refletem a cooperação mútua entre população e profissionais de saúde.

A Longitudinalidade é um fator essencial para o sistema de saúde, pois, permite que as pessoas tenham uma continuidade do atendimento à saúde e tende a produzir diagnósticos e tratamento mais precisos, além da redução de encaminhamentos desnecessários para especialistas e para a realização de procedimentos de maior complexidade ${ }^{10}$.

O valor alto do escore do atributo longitudinalidade, está relacionado ao reconhecimento pelos cuidadores de criança que o mesmo profissional está sendo responsável pelo acompanhamento da criança na ESF. Embora o escore deste atributo seja alto, os entrevistados dizem que os profissionais da ESF não conhecem os aspectos relativos às condições de trabalho da família. É relevante ressaltar, que apesar destas dificuldades, os entrevistados apresentam-se confortáveis para se expressarem nas consultas.

O atributo Coordenação é dividido em Coordenação - Integração de Cuidados e Coordenação - Sistema de Informações. Tal divisão foi realizada com o propósito de facilitar a compreensão dos resultados na íntegra, conforme sugestão do manual do PCATool ${ }^{8}$.

O atributo Coordenação - Integração de Cuidados obteve escore igual a 7,60, valor superior ao valor de referência. Apenas 5 (14,7\%) dos entrevistados responderam que "sim" a pergunta relacionada ao atributo Coordenação - Integração de Cuidados: "Sua criança foi consultar qualquer tipo de especialista ou serviço especializado no período em que ela está em acompanhamento na ESF?".

A coordenação é uma forma de continuidade por parte dos profissionais no reconhecimento de problemas. Quando se perguntou aos entrevistados se o profissional da ESF pareceu interessado na qualidade do cuidado que foi prestado a criança no especialista, 20\% respondeu que "provavelmente não" e 20\% respondeu que "com certeza não", o escore desta pergunta foi igual a 6, mostrando-se abaixo do valor de referência.

As considerações referentes aos registros de saúde ou boletins de atendimento (fichas de atendimento, carteira de vacinação) e o registro nos prontuários são tópicos avaliados no atributo Coordenação - Sistema de Informações. O atributo Coordenação - Sistema de Informações obteve escore igual a 8,89, alto em relação ao valor de referência 6,6.

O bom desempenho do atributo Coordenação - Sistema de Informações representa que os registros de saúde ou boletins de atendimento estão sendo instrumentos de comunicação entre profissionais e usuários do SUS.

$\mathrm{O}$ atributo Integralidade se divide em Integralidade - Serviços Disponíveis que é composto por tópicos que avaliam os serviços disponíveis na APS e pela Integralidade - Serviços Prestados que possui os itens que avaliam os serviços realmente prestados ${ }^{8}$. Esses itens são sobre toda a experiência do entrevistado com o serviço de saúde. O escore obtido com a Integralidade Serviços Disponíveis, foi igual a 6,78 e o escore obtido com a Integralidade - Serviços Prestados foi igual a 9,39, estes valores são superiores ao valor de referência.

No atributo Integralidade - Serviços Disponíveis, o indicador de disponibilidade de vacinas, ou seja, quando perguntamos da disponibilidade de vacinas na ESF para os entrevistados, $67,65 \%$ responderam que "provavelmente não", o escore obtido com este indicador foi igual a 1,76 , valor inferior ao valor de referência igual a 6,6. Esta situação é explicada pela inexistência de vacinas na unidade da ESF. O município de Quatá possui serviço de imunização que fica 
alocado e centralizado no centro de saúde que atende as quatro ESF.

A prevenção de enfermidades tem como objetivo a redução do risco de se adquirir uma doença específica por reduzir a probabilidade de que uma doença ou desordem venha a afetar um indivíduo ${ }^{11}$. Imunização é uma das atividades de prevenção primária que inclui promoção da saúde e proteção específica, constituindo-se assim em um dos principais componentes da APS $^{12}$.

Outra questão importante foi observada também no atributo Integralidade - Serviços Disponíveis no que tange o indicador de disponibilidade do serviço de sutura na ESF, 73,53\% dos entrevistados responderam que o serviço de sutura "provavelmente não" está disponível na ESF. No município de Quatá, o serviço de sutura não é oferecido pela ESF, mas está disponível no Ambulatório de Saúde Municipal. A sutura é um procedimento que de acordo com Starfield ${ }^{4}$ as unidades de APS devem fazer arranjos que possibilitem a sua realização.

O atributo Orientação Familiar tem a intenção de analisar, na visão do entrevistado, a importância que o profissional (médico e enfermeiro) dá para a opinião do cuidador da criança em relação à escolha do tratamento e cuidados da criança, e se o profissional faz perguntas sobre doenças que ocorrem na família como diabetes, hipertensão e outras doenças de influência genética. Nesse atributo obteve-se escore igual a 7,29, valor superior ao valor de referência.

Uma informação importante em relação ao atributo Orientação Familiar é que quando se perguntou aos entrevistados se "O profissional pergunta sobre suas ideias e opiniões?" obteve-se o escore igual a 6,08, valor inferior ao de referência de 6,6, isto demonstra que ainda ocorre a relação vertical entre os profissionais de saúde e os usuários do SUS, ou seja, os usuários do SUS pouco participam da escolha de seu tratamento. Este atributo é muito importante quando queremos avaliar a ESF, já que um dos pilares dessa estratégia é o foco na família.

No que tange o atributo Orientação Familiar, observou-se que quando foi perguntado aos entrevistados se "O profissional de saúde pergunta sobre suas ideias e opiniões sobre o tratamento e cuidado da criança?" , 17,6\% responderam "com certeza não" e 20,6\% responderam que "provavelmente não". Os profissionais de saúde ainda atuam com o modelo tradicional de educação em saúde em que o médico e o enfermeiro são detentores do saber enquanto que a população são seres passivos e que não participam da construção de seu projeto terapêutico e do projeto terapêutico de sua família.

O atributo Orientação Comunitária diz respeito ao conhecimento do contexto social onde as pessoas vivem. Uma das características da APS, é que a condição de saúde de uma comunidade é determinada pelas características ambientais, características comportamentais e o senso de conexão e de graus de coesão social na comunidade. Para que a APS aperfeiçoe a saúde, ela deve enfocar a saúde no meio social e físico no qual as pessoas vivem e trabalham ${ }^{12}$.

O escore obtido no atributo Orientação Comunitária foi igual a 7,72, valor superior ao valor de referência, no entanto, é de grande importância ressaltar que os profissionais da ESF pouco estimulam e convidam a população para a participação e controle social, esta situação é constatada ao perguntar aos cuidadores de crianças menores de dois anos se "O serviço de saúde convida os membros da família para participarem do Conselho Local de Saúde?" e obtém-se que 58,82\% dos entrevistados dizem que "com certeza não" e que 14,70\% dos entrevistados dizem que "provavelmente não", o escore desse indicador de participação social foi de 2,25, valor bem abaixo do valor de referência de 6,6.

O atributo Orientação Comunitária é importante quando se quer avaliar a ESF, pois seus itens incluem se os profissionais fazem a visita domiciliária, prática diferencial da ESF, e se os profissionais fazem pesquisa sobre o processo saúde doença da população e se conhecem o contexto de vida e saúde das famílias.

Nesse contexto é importante destacar que a participação social é uma importante ferramenta para empoderar as pessoas sobre seus direitos 
em relação à prestação dos serviços de saúde. Os profissionais que trabalham na ESF, por sua proximidade com a população, devem estimular a participação social e o controle social por parte da comunidade.

No presente estudo, a avaliação do serviço da ESF utilizando o instrumento PCATool, permitiu aferir a percepção dos cuidadores sobre a qualidade do serviço em relação a população infantil.

A perspectiva do usuário indica uma fonte de avaliação importante dentro do processo assistencial, pois é quem efetivamente experimenta o serviço em aspectos positivos e negativos. Ocasionalmente, esta pode ser uma visão mais crítica do que aquela realizada por outros atores sociais ${ }^{13}$.

Os escores de cada atributo da APS no geral foram satisfatórios, ressaltam-se os valores bem acima do valor de referência dos atributos "Grau de Afiliação", "Primeiro Contato - Utilização" e "Integralidade - Serviços Prestados". Isto demonstra que a ESF tem sido a porta de entrada do sistema de saúde para as crianças menores de dois anos que moram na área de abrangência da ESF estudada, e que apesar de não possuir os serviços de vacina e sutura, a ESF tem desenvolvido a maioria dos serviços de saúde que são preconizados para APS.

O Escore Essencial, escore obtido pela soma do escore médio dos componentes que pertencem aos atributos essenciais e do escore do Grau de Afiliação dividido pelo número de componentes, apresentou escore igual a 7,62 , valor superior ao valor de referência 6,6. O Escore Geral, escore obtido pela soma do escore médio dos atributos essenciais somados aos atributos derivados divididos pelo número total de componentes, apresentou escore igual a 7,60, valor superior ao valor de referência. Os Escores Essencial e Geral com valores satisfatórios revelam que a ESF estudada é considerada boa fonte de atenção à saúde na percepção dos cuidadores das crianças menores de dois anos.

Os resultados nos permite afirmar que, na percepção dos cuidadores, a assistência à saúde da criança no âmbito da unidade de ESF está de acordo com os princípios da APS, no entanto, os atributos Acesso de Primeiro Contato - Acessibilidade e Integralidade - Serviços Disponíveis precisam melhorar sua operacionalização na ESF.

A ESF estudada precisa melhorar a oferta de serviços com possíveis adequações em seu processo de trabalho para superar as dificuldades que os usuários têm de se obter uma consulta no mesmo dia, e mudar a relação entre profissional e cliente que segundo os entrevistados está pautada na comunicação horizontal, criando uma verticalização nas relações.

Crianças enfrentam inúmeros desafios ambientais e sociais nos contextos das famílias, escolas e comunidades que afetam significativamente os seus resultados de bem-estar e saúde. Para que os cuidados primários tenham uma abordagem ampla e resolutiva, é necessário que a APS tenha uma estrutura organizada, processo de trabalho inovador e materiais necessários, além de profissionais qualificados para resolver as necessidades de vida e saúde das crianças ${ }^{14}$.

Nessa perspectiva os pesquisadores Harzheim e Álvarez-Dartdet ${ }^{15}$ dizem que a efetividade da APS na assistência à saúde infantil, está diretamente relacionada com a prevenção de doenças e a promoção à saúde, ações que geralmente são desempenhadas no âmbito da ESF. Nesse contexto é importante dizer que a ESF possui ações que produzem mudanças significativas nos indicadores de saúde da criança.

\section{CONCLUSÃO}

Em relação à avaliação geral, vale ressaltar que o estudo abrange apenas a perspectiva dos cuidadores de crianças menores de dois anos que utilizam o serviço de ESF, podendo nesse caso, não representar a complexidade e a totalidade do serviço de saúde da criança disponível na APS do município de Quatá-SP. Nesta perspectiva vale ressaltar que os indicadores de saúde que mostram a qualidade de vida e saúde das crianças, bem como de acesso e qualidade dos serviços dispensados a este grupo vulnerável, permite o retrato da saúde municipal, já que as crianças devem ter a disponibilidade e a 
preferência para realização das ações prioritárias de saúde.

Neste estudo observou - se que os escores dos atributos da APS avaliados de uma forma geral foram satisfatórios, no entanto, os atributos Acesso de Primeiro Contato - Acessibilidade e Integralidade - Serviços Disponíveis precisam melhorar sua operacionalização a partir da reorganização do processo de trabalho que envolve a disponibilização de horários fora do horário comercial; maior facilidade para agendamento de consultas, atendimento imediato nas doenças agudas; reorganização e aquisição de estrutura e insumos para disponibilizar imunobiológicos, serviço de sutura e outros serviços nas unidades de saúde da família.

Um ponto positivo que esta pesquisa traz, é a frequência e a intensidade do uso da ESF como o primeiro serviço a ser procurado pelos usuários, mesmo quando há um novo problema de saúde ou ainda quando há a necessidade de encaminhamento para especialistas. A continuidade do atendimento avaliado por meio do atributo de Longitudinalidade está presente no processo de trabalho da ESF, o que é imprescindível para a resolutividade dos problemas de saúde e atendimento das necessidades de saúde.

Contudo, é valido ressaltar que novos esforços são necessários para melhorar a qualidade do serviço da ESF, para tal, é preciso repensar a partir dos resultados obtidos nos atributos a possibilidade desses esforços serem entendidos como fundamentais para novas mudanças comportamentais, organizacionais e estruturais no serviço, mantendo a responsabilidade no processo de trabalho de todos envolvidos.

\section{REFERÊNCIAS}

1. Brasil. Ministério da Saúde. Secretaria de Políticas de Saúde. Projeto da Saúde. As Cartas da Promoção da Saúde. Brasília; 2002.

2. Brasil. Ministério da Saúde. Secretária de Atenção em Saúde. Departamento de Atenção Básica. Manual do Instrumento de Avaliação da Atenção Primária à Saúde. Brasília; 2010.

3. Brasil. Ministério da Saúde. Secretaria de Atenção à Saúde. Departamento de Atenção Básica. Caderno de Atenção Primária - rastreamento. Brasília; 2010.

4. Contandriopoulos AP, Champagne F, Denis JL, Pineault R. A Avaliação na área da saúde: conceitos e métodos. In: Hartz ZMA, organizadora. Avaliação em saúde: dos modelos conceituais à prática na análise da implantação de programas. Rio de Janeiro: Editora Fiocruz, 1997. p. 29-47.

5. Czeresnia D, Freitas CM. organizadores. Promoção da saúde: conceitos, reflexões, tendências. Rio de Janeiro: Editora Fiocruz; 2003.

6. Donabedian A. The Quality of Care: How Can It Be Assessed?. JAMA [periódico na internet]. 1998 set [citado 2013 abr. 03]. Disponível em: http//jama.amaassn.org/contente/260/12/1743.full.pdf+html.

7. Ermel RC, Fracolli LA. O trabalho das enfermeiras no Programa de Saúde da família de Marilia/SP. Rev Esc Enferm USP. 2006;40(4):533-539.

8. Harzheim E, Álvarez-Dardet C. Evaluación de la atención a la salud infantil del Programa Saúde da Família en región de Porto Alegre, Brasil. 2007;3(9):60-61.

9. Instituto Brasileiro de Geografia e Estatística (IBGE) [homepage na internet]. Brasília; c2013-2014. [atualizado 2013 nov. 11; citado 2014 jan. 22]. Cidades. Disponível em: http://www.cidades.ibge.gov.br/xtras/home.php

10. Leão CDA, Caldeira AP, Oliveira MMC. Atributos da atenção primária na assistência à saúde da criança: avaliação dos cuidadores. Rev. Bras. Saúde Mater. Infant. 2011;11(3):323-334.

11. Novaes HMD. Avaliação de programas, serviços e tecnologias em saúde. Rev. Saúde Pública. 2000;34(5): 547-559.

12. Oliveira VBCA. Avaliação da atenção primária à saúde da criança no município de Colombo - Paraná [dissertação]. São Paulo: Escola de Enfermagem, Universidade de São Paulo; 2005.

13. Sousa MF. O Programa da família no Brasil: análise do acesso à atenção básica. Rev. Bras. Enferm. 2008;61(2):153-158. 14. Kuo AA, Etzel RA, Chilton LA, Watson C, Gorski PA. Primary Care Pediatrics and Public Health: Meeting the Needs of Today's Children. American Journal of Public Health. 2012;102(2):17-e23.

15. Starfield B. Atenção Primária: equilíbrio entre a necessidade de saúde, serviços e tecnologias. Brasília: Unesco/Ministério da Saúde; 2002. 